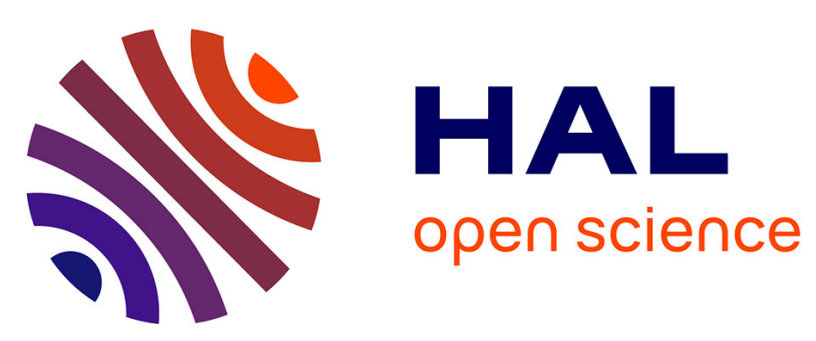

\title{
Assembly of Anisotropic Nanocellulose Films Stronger than the Original Tree
}

\author{
Rémi Merindol, Seydina Diabang, Randy Mujica, Vincent Le Houerou, \\ Thierry Roland, Christian Gauthier, Gero Decher, Olivier Felix
}

\section{- To cite this version:}

Rémi Merindol, Seydina Diabang, Randy Mujica, Vincent Le Houerou, Thierry Roland, et al.. Assembly of Anisotropic Nanocellulose Films Stronger than the Original Tree. ACS Nano, 2020, 14, pp.16525-16534. 10.1021/acsnano.0c01372 . hal-02997614

\section{HAL Id: hal-02997614 https://hal.science/hal-02997614}

Submitted on 30 Nov 2020

HAL is a multi-disciplinary open access archive for the deposit and dissemination of scientific research documents, whether they are published or not. The documents may come from teaching and research institutions in France or abroad, or from public or private research centers.
L'archive ouverte pluridisciplinaire HAL, est destinée au dépôt et à la diffusion de documents scientifiques de niveau recherche, publiés ou non, émanant des établissements d'enseignement et de recherche français ou étrangers, des laboratoires publics ou privés. 


\section{Assembly of Anisotropic Nano-Cellulose Films Stronger than the Original Tree}

Rémi Merindol, ${ }^{l}$ Seydina Diabang, ${ }^{,}$Randy Mujica, ${ }^{l}$ Vincent Le Houerou,,${ }^{1, \dagger}$ Thierry Roland, ${ }^{2}$ Christian Gauthier, ${ }^{l}$ Gero Decher ${ }^{1,3,4, *}$ and Olivier Felix ${ }^{1,4, *}$

${ }^{1}$ Université de Strasbourg, CNRS, Institut Charles Sadron UPR22, F-67000 Strasbourg, France.

${ }^{2}$ Université de Strasbourg, CNRS, INSA de Strasbourg, Institut Charles Sadron UPR22, F67000 Strasbourg, France.

${ }^{3}$ International Center for Frontier Research in Chemistry, F-67083 Strasbourg, France.

${ }^{4}$ International Center for Materials Nanoarchitectonics, Tsukuba, Ibaraki 305-0044, Japan.

Present Address: ${ }^{\dagger}$ Université de Strasbourg, CNRS, ICube UMR 7357, F-67412 Illkirch, France.

ABSTRACT: Natural structural materials frequently consist of multi-material nano-composites with complex superstructure giving rise to exceptional mechanical properties, but also commonly preventing access to their synthetic reproduction. Here we present the spin-assisted layer-bylayer assembly of anisotropic wood-inspired films composed of anionic cellulose nanofibrils and cationic poly(vinyl amine) possessing a tensile strength that exceeds the one of the wood from which the fibers originate. The degree of orientation of the nanofibrils was studied by atomic 
force microscopy and depends strongly on the distance from the center of the spun surface. The nanofibrils are preferentially aligned in the direction of the shear flow and consequently, the mechanical properties of such films differ substantially when measured parallel and perpendicular to the fibril orientation direction. For enabling a diversity of bio-inspired applications including sensing, packaging, electronics or optics, the preparation of nanocomposite materials and devices with anisotropic physical properties requires an extreme level of control over the positioning and alignment of nanoscale objects within the matrix material.

KEYWORDS: anisotropic films, spin-assisted LbL assembly, nano-reinforcement, woodinspired composites, cellulose nanofibrils, ultra strong materials

Biological nano-reinforced composites (e.g. bone, wood or nacre) possess sophisticated superstructures, organized hierarchically from the micro- to the macroscale via molecular assembly. ${ }^{1}$ This intricate architecture, optimized through evolutionary selection, grant these natural materials with sought-after combinations of properties such as strength and toughness above that of their individual constituents. An interesting example is natural wood which combines lightweight, strength and flexibility properties that are sought for mechanical engineering (Figure 1A). These exceptional mechanical properties emerge from the hierarchical and anisotropic architecture of wood where strong cellulose nanofibrils wrap helically in multiple layers around the wood cell walls.,3 Such nano- and micro-scale organization of the cellulose fibers minimizes weight and maximizes the tensile strength and modulus where it is most needed, that is typically parallel to the grain of the wood, while maintaining flexibility 
perpendicular to the grain direction provides flexibility to the structure (Figure 1A). At an even finer scale the helical pitch also decreases with the age of the tree, providing more flexibility to young trees and more tensile strength to old ones. ${ }^{4}$ While nature masters the organization of anisotropic nano-objects like nanocelluloses into complex superstructures, the development of synthetic nano-composite materials with complex and precisely controlled architectures has proven to be difficult due to the lack of suitable approaches for their preparation. The improvement of mechanical properties of a synthetic material is typically carried out by preparing (nano-)composites by dispersing strengthening fillers within a polymer matrix.5,6 Nevertheless, the components of such composites tend to phase separate, which highly affects the "reinforcing effect" of the material that is dependent on distances between the strengthening agents. ${ }^{7,8}$
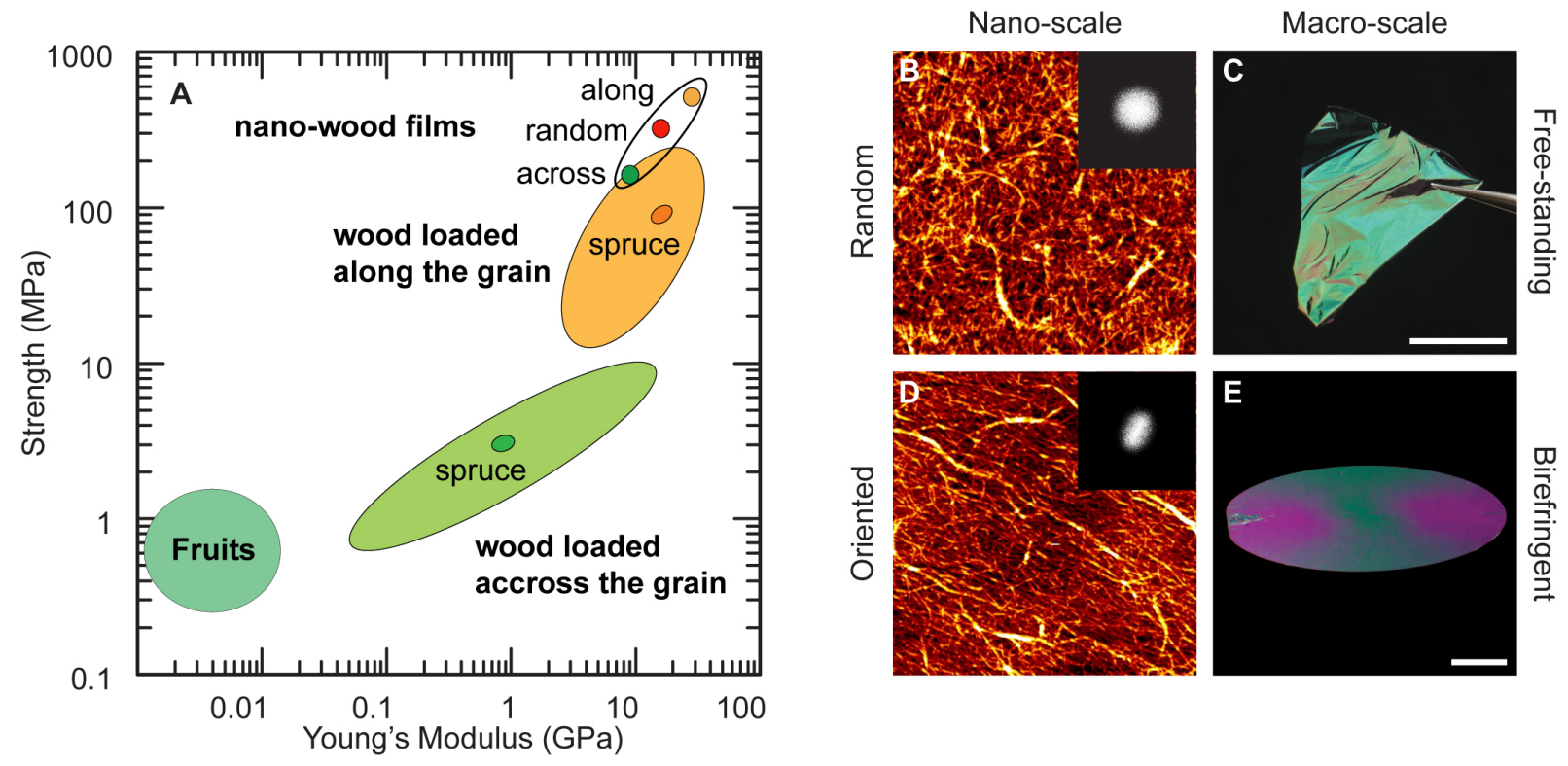

Figure 1. Orientation in natural and synthetic composites. (A) Young's modulus plotted against maximal strength for natural plant based materials and our oriented thin composite films. ${ }^{2}$ 
Nanoscale organization of the cellulose fibrils in films assembled by dip- (B) and spin-assisted LbL assembly at $10000 \mathrm{rpm}$ (D) as observed by AFM. Insets represent the Fourrier transform of the corresponding AFM images. The dimensions of the images are $3 * 3 \mu \mathrm{m}$ and the height range $15 \mathrm{~nm}$. (C) Macroscale aspect of a free standing film prepared by spin-assisted assembly, the green shade comes from optical interferences from light reflected at the two interfaces of the film. (E) Under grazing incidence observation two different interference colors appear due to the difference of refractive index parallel and perpendicular to the cellulose fibrils (Maltese Cross, see Supporting Information). Scale bars in $\mathrm{C}$ and $\mathrm{E}$ are $1 \mathrm{~cm}$ respectively.

In the context of a global environmental crisis bio-sourced composite materials based on nanocellulose derivatives (cellulose nanocrystals (CNC), bacterial cellulose and cellulose nanofibrils (CNF)) exhibiting valuable physical properties (outstanding mechanical strength, low density, biodegradability and biocompatibility) are gaining importance as alternatives to fully synthetic composites, in fields comprising e.g strong nano-composites, gas barrier coatings and hydrogels for medical applications. ${ }^{9,10}$

CNF-based composites are generally prepared by casting, ${ }^{11}$ vacuum filtration, ${ }^{12}$ and wet spinning, ${ }^{13,14}$ however the strongly anisotropic physical properties of individual fibrillar cellulosic nano-objects are often averaged out and cannot be exploited in disordered multi-particle systems. While wet spinning can generate oriented filament structures, ${ }^{13}$ film preparation techniques require a subsequent hot drawing ${ }^{15}$ or wet stretching ${ }^{12}$ step in order to align the cellulose nanofibrils. Such macro-scale post processing limits the preparation of hierarchical structures with multiple directions of orientation similar to the one found in wood structures. ${ }^{16}$ With respect to the preparation of multifunctional nano-organized composite materials with a well-controlled 
positioning of its constituents, the layer-by-layer (LbL) assembly, ${ }^{17}$ is probably the most simple and versatile nanofabrication method. This bottom-up approach involves the alternate deposition of cationic and anionic components (polyelectrolyte, nanoparticles, ...) on a charged surface to form a multi-material thin film with controlled organization and defined properties at the nanoscale. The structure and properties of LbL films are defined by the composition, the film structure and the experimental conditions used for the build-up. The fact that LbL-assembly is based on attractive interactions between adjacent layers helps to prevent or at least limit the phase separation of the polymer matrix and of the strengthening agent, at least with respect to linearly growing films whose components are typically trapped during deposition. LbLassembly ${ }^{17}$ is already well established as a readily accessible technique for the preparation of multicomponent architectures with exceptional mechanical properties., ${ }^{5,18}$ Among suitable components for incorporation in LbL-based multilayer systems with enhanced physical properties, nanocelluloses appear as highly attractive candidates. Both types of nanocelluloses (CNFs and CNCs) were successfully used to prepare LbL-films ${ }^{22-25}$ and have also been oriented in suspensions and in thin films by applying external fields (magnetic, electrical, or mechanical). ${ }^{26-32}$ Among all methods available for aligning anisotropic nano-objects like nanocelluloses on surfaces directed assembly by shear flow seems to be the best suited as it requires less heavy equipment and allows to cover large surface areas $\left(\right.$ several $\left.\mathrm{cm}^{2}\right)$. Cranston et al. showed the spin-assisted assembly of CNC-based LbL films with a radial orientation and optical properties varying from the center (isotropic) to the edge (anisotropic) of the samples. ${ }^{33,34}$ We also reported recently the LbL-assembly of multi-property films composed of non-oriented $\mathrm{CNFs},{ }^{19}$ as well as the preparation of thin unidirectionally oriented CNF-based LbL films with anisotropic optical properties by grazing incidence spraying (GIS). ${ }^{35}$ Unfortunately, for the 
present work on mechanical properties, anisotropic CNF films prepared by GIS are not (yet) suitable as GIS films tend to be too brittle owing to defects that promote film rupture, especially in the case of micron thick films.

In the present article we report the fabrication and mechanical properties of anisotropic woodinspired nanostructured films constituted of CNFs and poly(vinyl amine) (PVAm) assembled by spin-assisted LbL-assembly. The latter allows the fast build-up of micron-thick CNF-based LbL films with a radial orientation on a large scale, a prerequisite for tensile strength measurements (Figure 1B-E). As expected, the deposition of CNF-multilayers turned out to be influenced by the deposition method (dipping versus spin-assisted assembly) and by the applied rotation speed during deposition. Surprisingly, thicker films were obtained by dipping than by spin-assisted assembly. Optically transparent CNF-based films assembled by dip- and spin-assisted assembly were detached from hydrophobizied substrates, and their mechanical properties were investigated depending on the deposition method, the rotation speed and the orientation of CNFs with respect to the direction of the applied mechanical stress. As expected the tensile strength and modulus increase from oriented films measured perpendicular to the fibril direction (highest flexibility) to random orientation and reach their maximum for oriented films measured parallel to the fibril direction (highest stiffness). Yet in all cases the mechanical properties obtained surpass those of most wood structures and more specifically spruce, the wood from which the nanofibrils used here are extracted (Figure 1A). ${ }^{2}$ In the direction of orientation the strength (490 $\mathrm{MPa}$ ) and Young's modulus (33 GPa) of the films match the ones of wet stretched films made of pure $\mathrm{CNF},{ }^{12}$ or PEG-grafted-CNF, ${ }^{36}$ but surpass those of wet stretched CNF-composites, ${ }^{12}$ or wet spun-filaments made of pure cellulose..$^{13}$ In comparison entirely synthetic nano-composites based on carbon-nanotubes and aramid fibers reach similar mechanical properties. ${ }^{37}$ In addition to 
shortening the time required to prepare ultra-strong highly ordered multilayers, ${ }^{38,39}$ spin-assisted LbL-assembly enables the preparation of complex hierarchical nanostructures.

\section{RESULTS AND DISCUSSION}

Assembly of thick non-oriented and oriented multilayers. The preparation of micron-thick LbL films was carried out by conventional dipping and spin-assisted assembly from a solution of cellulose nanofibrils (CNF) and a solution of poly(vinyl amine) (PVAm) both dissolved in ultrapure water at a concentration of 0.9 and $1 \mathrm{~g} / \mathrm{L}$ respectively. The $\mathrm{pH}$ of $\mathrm{CNF}, \mathrm{PVAm}$ and rinsing solutions was adjusted at 6,8 and 6 respectively prior to assemble the LbL films on silicon wafers. Figure $2 \mathrm{~A}$ presents the thickness evolution of the multilayer with the increasing number of deposition cycles (up to 200 layer pairs). We observe a higher thickness increment per layer-pair (LP) for films assembled by dipping $(9 \mathrm{~nm} / \mathrm{LP})$ than those prepared by spin-assisted assembly at $10000 \mathrm{rpm}(7 \mathrm{~nm} / \mathrm{LP})$ and $8000 \mathrm{rpm}(6 \mathrm{~nm} / \mathrm{LP})$. Despite the lower thickness increment observed for films prepared by spin-assisted assembly compared to dipping the gain in time to prepare thick film is tremendous. The minimum time required to build a $1 \mu \mathrm{m}$ thick film decreases from 35 hours with conventional LbL-assembly by immersion to about 5 hours using spin-assisted assembly at $8000 \mathrm{rpm}$. We assume that for films containing highly anisotropic nanoparticles, rinsing in the presence of high shear forces promotes the desorption of weakly bound nanofibrils that would not be rinsed off in the case of dip-assisted LbL. One should also note that the dipped films were prepared by immersion of the substrate in the CNF or PVAm solution for 5 min rather than few seconds..$^{38}$ This fact alone could explain the growth difference for CNF-based films assembled by dip- and spin-assisted assembly. The slight increase of 
thickness observed for films assembled by spin-assisted assembly at higher speed could be attributed to an increased packing density of nanofibrils with higher shear forces. This phenomenon was already observed at the center of samples where the LbL films are about 2-3\% thinner than at the edges due to lower applied shear forces. ${ }^{38}$ The Figure 2B-E shows the interference colors of (PVAm/CNF) films with an increasing thickness prepared by spin-assisted assembly at $8000 \mathrm{rpm}$. These colors are "structural colors" which are characteristic of thin films with uniform thickness and refractive index. The homogeneity of the color over most of a sample is evidence for the microscale precision of the film thickness, the center of the sample being slightly thinner than the outer regions. The homogeneity of the films at the microscale is also apparent on the Atomic Force Microscopy (AFM) images used for the thickness measurements Figure 2F.
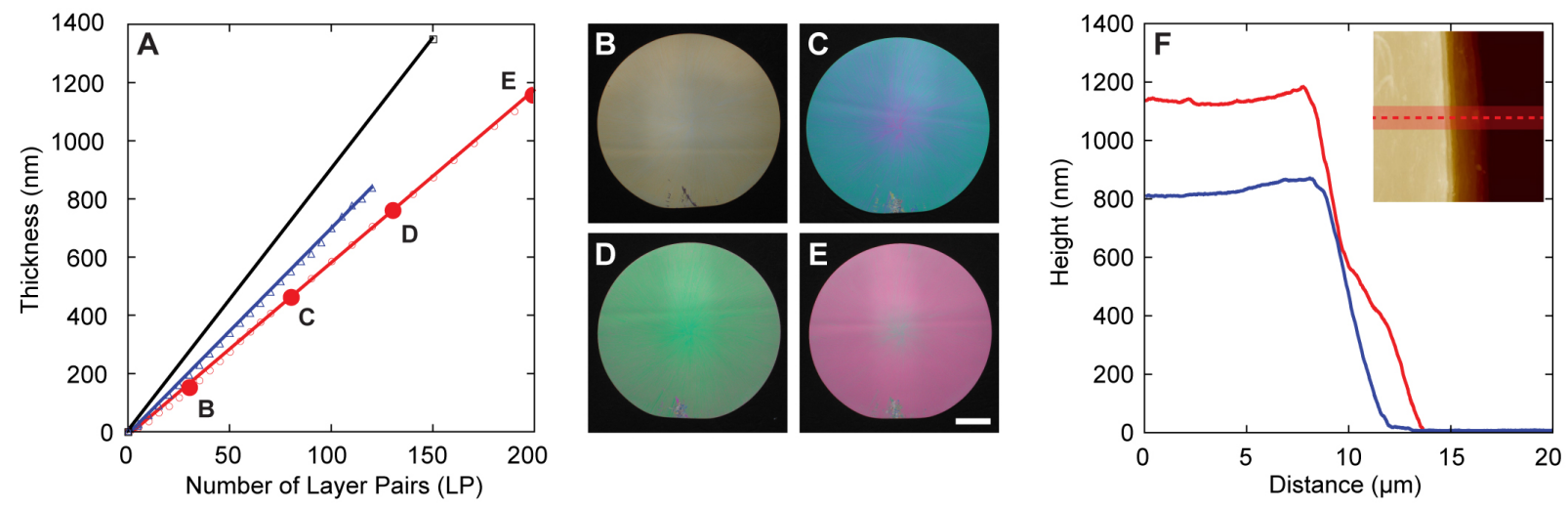

Figure 2. Growth, thickness and homogeneity of CNF-based films prepared by spin-assisted assembly. (A) Assembly of (PVAm/CNF) $)_{n}$ LbL films by dipping (black) and spin-assisted assembly at $8000 \mathrm{rpm}$ (red) and $10000 \mathrm{rpm}$ (blue) followed by ellipsometry. The larger red dots correspond to the photographs B to E. Error bars are smaller than the symbol size. (B), (C), (D) 
and (E) Optical images of (PVAm/CNF) films with an increasing thickness $(180,470,760$ and $1150 \mathrm{~nm}$ respectively) prepared by spin-assisted assembly at $8000 \mathrm{rpm}$. The film is still on its silicon wafer support and the color comes from the reflection of a white background viewed close to the normal of the sample. The scale bar for B-E images is given in $\mathrm{E}$ and corresponds to $1 \mathrm{~cm}$. (F) Height profiles extracted from AFM images of the edge of oriented films assembled by spin-assisted assembly at $8000 \mathrm{rpm}$ (red) and $10000 \mathrm{rpm}$ (blue) on a silicon substrate. The inset shows an AFM image for a film prepared by spin-assisted assembly at $8000 \mathrm{rpm}$ where the red line indicates the width and direction of the height profile. The dimensions of the inset are $20 * 20$ $\mu \mathrm{m}$ and the height scale $2 \mu \mathrm{m}$.

While the use of more advanced apparatus (generalized ellipsometry or Müller matrix polarimetry) is required for determining accurately the optical constants of anisotropic materials, we used here a routine ellipsometer and a fixed refractive index to estimate the thickness of our films despite of their anisotropy, an approach that has already been demonstrated to be practical and sufficiently precise. ${ }^{35}$ Cellulose nanocrystals are anisotropic objects known to present a different refractive index parallel and perpendicular to their long axis. ${ }^{33}$ The small difference between these two values allowed us to set the refractive index to 1.55 in all our measurements and to obtain reasonable results based on this assumption. The deviation between positions where the nanofibrils were oriented parallel or perpendicular to the plane of incidence was below $1 \%$ of the total thickness above $500 \mathrm{~nm}$. The final thickness of these anisotropic films measured by ellipsometry was confirmed by imaging the edge of supported films with AFM, e.g. for the film assembled at $8000 \mathrm{rpm}$ we obtained $1.15+/-0.01 \mu \mathrm{m}$ by ellipsometry and $1.13+/-0.03 \mu \mathrm{m}$ by AFM (Figure 2F). The effect of the fiber orientation on the refractive index is however visible 
by eyes under grazing incidence. The different refractive indexes parallel and perpendicular to the fiber axis generate the green and pink shape visible in Figure 1E (reminding of the Maltese Cross).

The mechanical characterization using tensile apparatus requires free-standing micron-thick multilayers. In order to obtain free-standing samples, we used hydrophobically modified silicon wafers. ${ }^{40,41}$ This approach allows building multilayers that adhere weakly on their substrate and can later be peeled off using a piece of tape or tweezers (Figure 1C). Hydrophobic surfaces are known to impact the adsorption of the first layers of polyelectrolytes but as previously reported these effects are negligible after the deposition of few layer pairs. ${ }^{42}$ After peeling the freestanding micron thick film is mechanically strong and flexible, yet completely transparent and displays a green color coming from the interference of light between the two surfaces of the film.

Characterization of CNF alignment in spin-assisted layer-by-layer assembly. As indicated previously, the orientation of the cellulose fibrils in the multilayers can be easily observed by eyes under grazing incidence (Figure 1E), yet more quantitative measurements are challenging. Due to the sensitivity of ellipsometry and reflectometry to the porosity, roughness and thickness of the multilayer, their use to determine the degree of orientation of the multilayer requires angle dependent measurements and extensive analysis..$^{43}$ Therefore we imaged the films using AFM and characterized the degree of orientation of the nanofibrils using OrientationJ, an ImageJ plugin that allows pixel-based characterization of the orientation (Figure 3).$^{44}$ Each pixel is colorcoded according to the alignment direction (Figure 3C). 

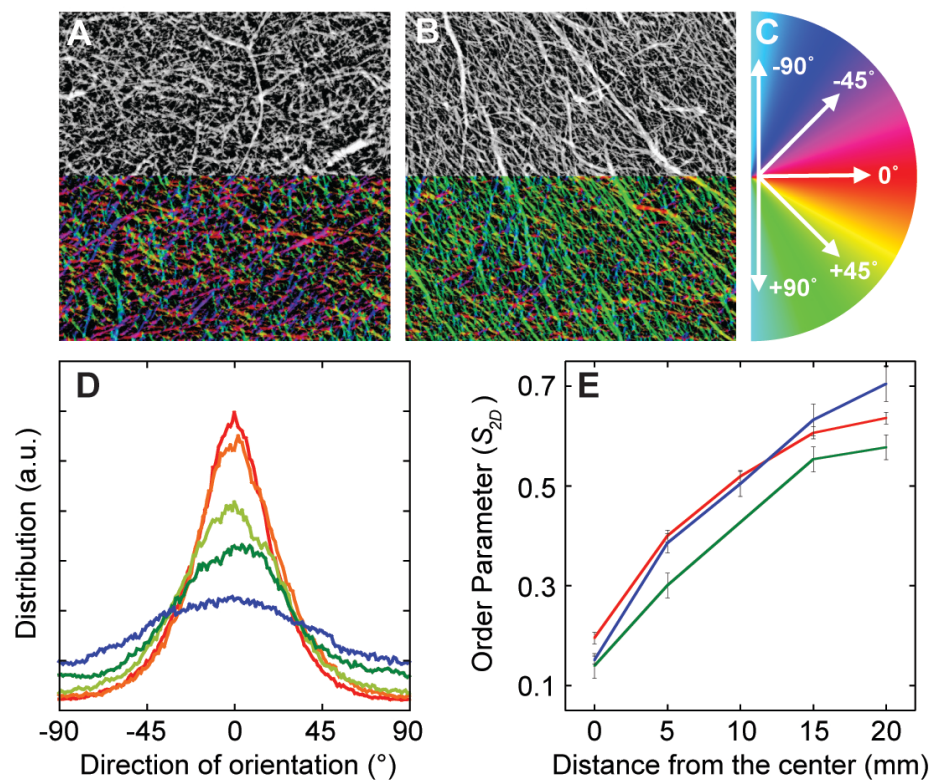

Figure 3. Characterization of CNF alignment in spin-assisted assemblies. (A) and (B) AFM images $\left(3 * 3 \mu \mathrm{m}\right.$ and a $15 \mathrm{~nm}$ range for the grey scale) of $(\mathrm{PVAm} / \mathrm{CNF})_{1}$ with the original black and white image (top) and the colorized output from OrientationJ (bottom). (C) Color-code with respect to the alignment direction used for images B and C. (D) Representative distribution of orientation measured at increasing distances from the spin axis of samples ( $0 \mathrm{~mm}$ (blue), $5 \mathrm{~mm}$ (dark green), $10 \mathrm{~mm}$ (light green), $15 \mathrm{~mm}$ (orange), and $20 \mathrm{~mm}$ (red)) prepared by spin-assisted deposition at $8000 \mathrm{rpm}$. (E) Order parameter calculated from AFM images, according to Equation 1, for samples prepared by spin-assisted assembly at 5000 (green), 8000 (red) and $10000 \mathrm{rpm}$ (blue) as function of the position of the imaged area from the center to the edge.

The software produces a visual representation of the degree of orientation of the nanofibrils as well as a quantitative measurement of the orientation distribution. The quantification of orientation based on the calculation of the structure tensor in a local neighborhood allows 
characterizing branched and curved structures such as the ones observed in our materials. This allows visualizing the general direction of orientation (main color) and the degree of alignment (color intensity) of the AFM images which can be characterized by the $2 \mathrm{D}$ order parameter $\left(S_{2 D}\right)$ calculated using Equation 1. We performed the quantification of orientation on AFM images of single layer structures as the flat and homogenous silicon support facilitates the analysis. ${ }^{45}$ Since each layer of nano-fibrillated cellulose is deposited on a layer of poly(vinyl amine) we assume that the orientation of the cellulose nanofibrils through the film is sensibly similar to what happens on the first layer. This assumption was confirmed by analyzing the angular variation of the in-plane relative brightness of unidirectionally aligned micron thick films by crossed polarized optical microscopy (Figure S1).

$S_{2 D}=\left\langle 2 \operatorname{Cos}(\theta)^{2}-1\right\rangle \quad$ (Equation 1$)$

where $\theta$ is the angle between each CNF main axis and the main direction of orientation of the sample. The $S_{2 D}$ value varies between 0 for randomly distributed CNFs (isotropic films) and 1 for perfectly aligned $\mathrm{CNFs}\left(\theta=0^{\circ}\right.$, anisotropic films $)$.

Typically one layer pair of PVAm and CNF was deposited on a freshly activated silicon wafer ( $5 \mathrm{~cm}$ in diameter). AFM images were taken every $5 \mathrm{~mm}(+/-0.5 \mathrm{~mm})$ from the center to the edge. Specific care was taken to avoid artifacts due to drift during imaging (scanning up and scanning down images were systematically compared). Typical images obtained at the center and near the edge of the sample are presented in Figure 3A,B respectively, with the corresponding color scale (Figure 3C). From the color-coded images it appears already clearly that CNFs are randomly distributed at the center (Figure 3A) while they are oriented at the edge (Figure 3B). In addition to illustrating the large difference of orientation along the diameter of the sample, this 
image analysis allows to control the quality of the orientation measurements. The orientation distribution under assembly at $8000 \mathrm{rpm}$ is presented in Figure 3D. As expected the orientation of the cellulose nanofibrils increases from the center to the edge. Surprisingly we observed some degree of orientation for most images taken at the center of the rotating substrate. We assume that the shear forces associated to the drop spreading during deposition and the probable nonsuperposition of the center of rotation with the drop falling point are responsible for this phenomenon. The order parameters for the samples prepared at 5000, 8000 and $10000 \mathrm{rpm}$ are reported in Figure 3E. In all cases the order parameter increases from the center to the edge while the maximal degree of orientation of the CNFs in the film also increases with rotation speed. At $2 \mathrm{~cm}$ from the center the order parameters measured were $0.58,0.63$ and 0.70 for sample prepared at 5000, 8000 and $10000 \mathrm{rpm}$ respectively. These values are comparable to the highest degree of orientation achieved by cold-drawing with a drawing ratio of 1.6 (i.e. 0.72). ${ }^{12} \mathrm{We}$ focused our characterization on the mechanical properties of films prepared at 8000 and 10000 as their higher orientation should lead to higher modulus and strength at break.

Mechanical properties of thick oriented LbL films. The tensile properties of anisotropic nanocomposites were measured parallel and perpendicular to the direction of orientation and compared with isotropic composites prepared by dipping (Figure 4). All studied films showed stress-strain curves with a typical shape (Figure 4C) and the corresponding data are presented in Table 1. The true values of stress and strain were achieved thanks to digital image correlation method as reported in the "methods" section. 

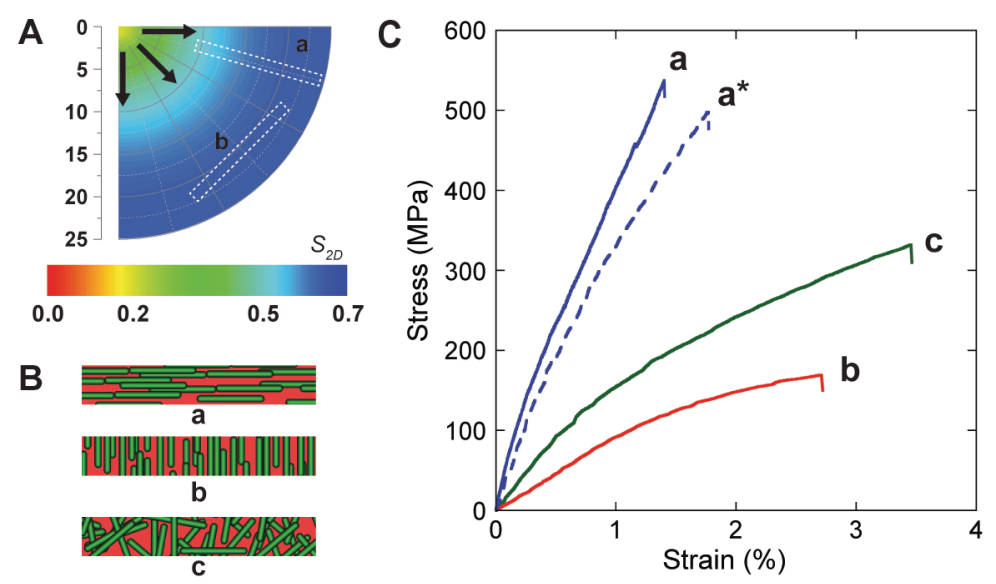

Figure 4. (A) Sketch showing the preparation of oriented sample (parallel (a) and perpendicular (b) to the CNF alignment) for mechanical tests from films prepared by spin-assisted assembly. The black arrows show the direction of orientation of the nanofibrils and the color-code the global orientation of CNFs on the sample. (B) Simplified sketch of the relative organization of the CNFs in oriented (a,b) and random (c) samples. (C) Stress-strain curves measured for thick CNF-based multilayers prepared by dipping (c) and spin-assisted LbL assembly at $8000\left(a^{*}, b\right)$ and $10000 \mathrm{rpm}(\mathrm{a})$.

Table 1. Mechanical properties obtained for thick $(\mathrm{PVAm} / \mathrm{CNF})_{\mathrm{n}}$ multilayers prepared by dipping (random) and spin-assisted LbL-assembly at 8000 and $10000 \mathrm{rpm}$ (oriented). The Young's modulus was measured on the first $0.3 \%$ of the curves. At least three measurements where done for each films in each direction.

\begin{tabular}{cccc}
\hline Sample & $\begin{array}{c}\text { Young's } \\
\text { Modulus } \\
(\mathrm{GPa})\end{array}$ & $\begin{array}{c}\text { Stress at } \\
\text { Break (MPa) }\end{array}$ & $\begin{array}{c}\text { Strain at } \\
\text { break (\%) }\end{array}$ \\
\hline $\mathbf{a}$ & $33.1(+/-2.0)$ & $473(+/-28)$ & $1.7(+/-0.7)$ \\
$\mathbf{a}^{*}$ & $32.8(+/-1.7)$ & $491(+/-19)$ & $2.0(+/-0.1)$ \\
$\mathbf{c}$ & $14.2(+/-1.0)$ & $286(+/-47)$ & $3.2(+/-0.8)$ \\
\hline
\end{tabular}


As expected, the Young's modulus and the tensile strength increase from oriented films measured perpendicular to the fibril direction (b) to random films (c) and to oriented films measured parallel to the fibril direction $\left(a, a^{*}\right)$. Cellulose nanofibrils are the reinforcing components at the origin of the exceptional mechanical properties of wood; their tensile modulus has been estimated at $100 \mathrm{GPa}$ and their failure strength above $1 \mathrm{GPa}^{2}$ In our case poly(vinyl amine) acts as a soft matrix which binds the fibers together and enables stress distribution, a role similar to the ones of lignin and hemicellulose in natural wood. When the cellulose fibrils are oriented perpendicularly to the solicitation most of the deformation occurs in the PVAm. Despite the weak mechanical properties of PVAm, the Young's modulus of the oriented films measured perpendicular to the fibril direction remains relatively high (9 GPa) mainly because the tests were performed at low humidity where the deformation of the dry poly(vinyl amine) is limited. As previously reported, increasing humidity would reduce both the Young's Modulus and the tensile strength of the polymer. ${ }^{19,46}$ On the other hand when the nanofibrils are oriented parallel to the direction of solicitation they bear most of the stress and films reach a modulus of $33 \mathrm{GPa}$ and a tensile strength of $490 \mathrm{MPa}$. As the modulus of cellulose nanofibrils is only weakly affected by humidity, the samples measured at higher humidity conditions had similar modulus, but the maximal stress decreased to about $300 \mathrm{MPa}$ because of the plasticizing effect of water that lowers the adhesion between the polymer matrix and the reinforcing fibers. ${ }^{19}$

According to composite theory, one could consider the expression of parallel and perpendicular extremum of Young's modulus values reported in Equations 2 and $3 .{ }^{47}$ Such simple model enable to decompose the composite moduli ( $E_{/ /}$and $E_{\perp}$ ) only based on the 
components modulus ( $\left.\mathrm{E}_{\mathrm{CNF}}, \mathrm{E}_{\mathrm{PVAm}}\right)$ and volume fraction of CNF named $f$. The CNF contend has been estimated to about $90 \%{ }^{19}$ which matches that of a compact cylinder arrangement, as expected due to the flexibility of the cellulose fibers that form dense mat as they dry. We can therefore estimate the Young's modulus of each component of the composite can be deduced by solving the resulting 2-variable system.
$E_{\|}=f * E_{C N F}+(1-f) * E_{P V A m}$
(Equation 2)
$E_{\perp}=\frac{1}{\left(\frac{f}{E_{C N F}}+\frac{(1-f)}{E_{P V A m}}\right)}$
(Equation 3)

It leads to the estimation of Young's moduli for both PVAm matrix phase of about $1.2 \mathrm{GPa}$ and cellulose fibers of about $36 \mathrm{GPa}$. On the one hand, we confirmed the value obtained for the PVAm matrix by nano-indentation conducted besides on micrometer-thick films. On the other hand the CNF modulus calculated falls below that measured on single fibers in the range of 120 to $167 \mathrm{GPa}, 2,48-52$ but matches the values found for cellulose network (e.g. nano papers) with an in-plane Young's modulus in the range 7 to $35 \mathrm{GPa} .^{2,53-56}$ Although the approach could be refined, e.g. using more advanced model knowing additional parameters on the PVAm phase, ${ }^{57}$ the composite theory appears relevant to estimate the Young's modulus of the CNF assembly and of the polymer matrix. Moreover, since the present materials consist of a rich fiber network fully aligned drown in PVAm, the $36 \mathrm{GPa}$ value may represent an upper-bound of elastic property associated to a nano-paper made of these CNFs. Indeed, this value belongs to the largest range of literature evoked previously. We believe that the discrepancy between single fibers and network modulus originates from the fibrillation process. This process decreases the average modulus of CNF compares to that which are measured on single crystalline fibers. Finally, these considerations may suggest that the role of PVAm is of minor importance in terms of mechanics, 
although it is crucial in the film building process. As expected the mechanical properties of randomly oriented films falls between the one of the oriented films solicited parallel and perpendicular to the fiber direction. The modulus reported here (14.2 GPa) is lower than the one expected according to Equation 4 for random composite $(19 \mathrm{GPa})$ given the parallel $\left(E_{/ /}\right)$and perpendicular $\left(E_{\perp}\right)$ modulus found in the oriented samples ${ }^{43}$ We believe that it may be due to a porosity prone to appear during processing the random network therefore inducing a bias compared to the previous estimation.

$E_{2 D}=\frac{3}{8} E_{\|}+\frac{5}{8} E_{\perp}$ (Equation 4)

Spin-assisted LbL assembly enables the preparation of highly anisotropic nanocellulose-based LbL films whose mechanical properties exceed that of the wood of the natural tree from which they are obtained and match that of the best cellulose-based materials. In spruce, the original tree, the tensile strength reaches $1 \mathrm{MPa}$ perpendicular and $90 \mathrm{MPa}$ parallel to the grain while the Young's moduli are 1 and $10 \mathrm{GPa}$ respectively. With a Young's modulus of about $33 \mathrm{GPa}$ and a tensile strength of up to $490 \mathrm{MPa}$ parallel to the alignment direction and values of $9 \mathrm{GPa}$ and of up to $145 \mathrm{MPa}$ respectively perpendicular to the alignment direction, the mechanical strength of the films is several times higher than the one of the specific natural wood (spruce) and the inplane mechanical anisotropy has a value of about 3.5 and reflects well its structural anisotropy. LbL films reach such an exceptional stiffness by increasing the cellulose content while maintaining high anisotropy compare to natural wood. It is believed that the mechanical properties of wood are a consequence of the hierarchical and anisotropic arrangement of cellulose fibrils within the different layers of the wood cell wall (Figure 5A). The latter is 
arranged in four discrete layers, an outermost primary layer composed of randomly distributed cellulose microfibers surrounding a secondary cell wall divided in three consecutive layers (S1, S2 and S3) containing cellulose microfibrils organized in different helical structures. All the layers are made of the same constituents (mainly cellulose, hemicellulose and lignin) but their composition and mechanical behavior varies significantly. While the primary layer is mostly composed of lignin (around 70\%) the S2 layer contains the highest content of cellulose (roughly $50 \%$ ). The S2 layer represents about $70-80 \%$ of the thickness of the cell wall and is the most important with respect to the mechanical strength of the cell, especially in the axial direction. ${ }^{2}$

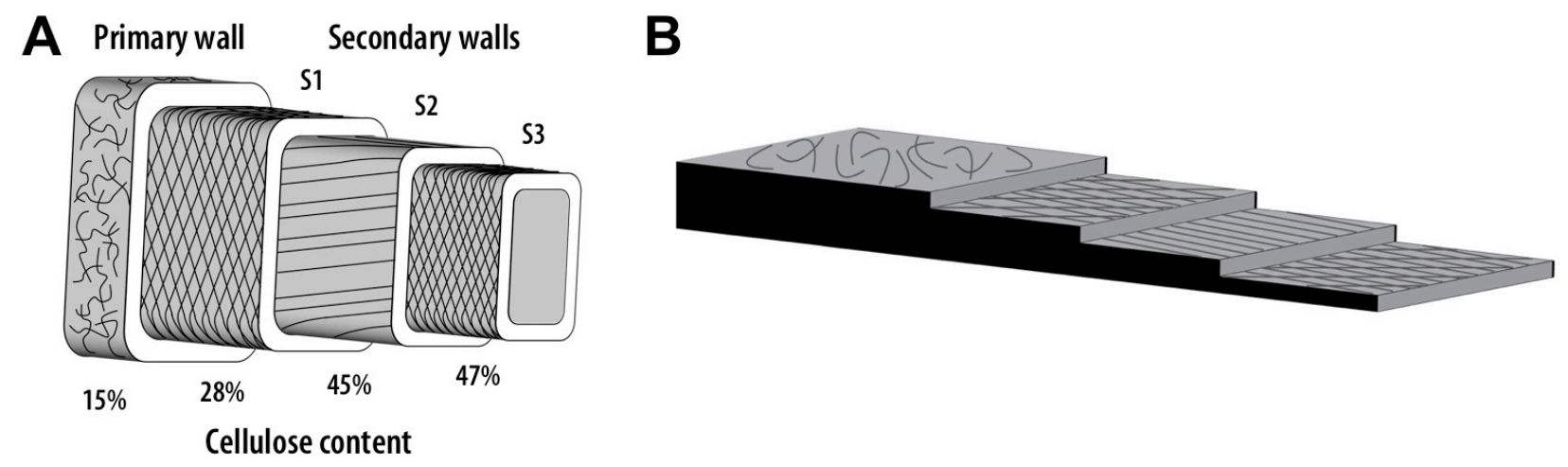

Figure 5. Sketch of the structure of the wood cell wall (adapted from Gibson²). (B) Depiction of a multilayer film mimicking the wood cell wall shown in A which is composed of four layers with cellulose nanofibrils arranged in different orientation in each layer.

We have already reported how the cellulose content of disordered CNF/PVAm films influences its mechanical properties and we are describing now how CNF densities and the alignment of CNFs contribute to markedly improved mechanical properties. As a result, the films 
discussed here exceed the mechanical strength of the wood from which the fibers were originally prepared and also compete with the best de-lignified and densified wood recently reported ${ }^{58,59}$ or crosslinked fibers based on native lotus cellulose fibers after glutaraldehyde crosslinking. ${ }^{8}$ These materials use native cellulose, without fibrillation step, which is an advantage to reach high strength, as mechanical fibrillation breaks the cellulose crystals and reduces their final strength. Yet the above processing steps offer very limited room to design, tune and optimize the nanoscale structure and composition of the films. Contrary to these classic processing methods above, the architecture of the films described here is brought about by a precise multistep process with compositional control at the molecular scale and which additionally allows for the in-plane alignment of fibrillar nano-objects in each layer.

The advantage of going through the fibrillation and reassembly steps is therefore that these steps enable a tunable and modular design of the final material. Recently, inspired by the structure of the S2 layer of the wood cell wall, Mittal et al. ${ }^{60}$ reported the flow-assisted assembly of cellulose nanofibrils in micron-sized filaments with record mechanical properties in the fiber direction (Young's modulus up to $86 \mathrm{GPa}$ and tensile strength up to $1.6 \mathrm{GPa}$ after cross-linking) matching the estimated theoretical limits reported for a single nanofibril (Young's modulus in the range of 120 to $167 \mathrm{GPa}$ and tensile strength above $1 \mathrm{GPa}){ }^{2,48-52}$ These results underline the apparent importance of efficient stress transfer from the macroscale filament to individual nanofibrils. Here it is interesting to see that the order parameter reported for hydrodynamically aligned filaments composed of long nanofibrils were similar (about 0.7) to the one obtained from our films obtained by spin-assisted assembly.

For testing how the complex superstructure of wood contributes to its observed mechanical properties one needs to evaluate the individual elements of its superstructure in isolated form and 
also in different superstructures built by combining these elements in different sequence. The preparation of a multilayer architecture comparable to the one observed in the cell wall of wood demands an approach that permits the controlled stacking of layers with well-defined in-plane orientation (random, unidirectional, helical) in different strata each of which may be a LbLmultilayer (Figure 5B). A common problem with hydrodynamic alignment and cold drawing is that the hierarchical structure of the materials is difficult if not impossible to vary by alternating for example layers of unidirectional and random alignment as in the wood cell wall and to prepare more complex anisotropic materials (e.g. criss-cross, helical) over large areas.

In contrast and in addition to providing a very high degree of orientation and mechanical strength, spin-assisted orientation has a broad potential for the preparation of composites with additional components (multi-materials) and with more complex superstructures. The latter is due to the fact that conventional, spin-assisted and spray-assisted LbL-assembly can freely be combined which allows to hierarchically construct multi-composite materials possessing multiple orientations. ${ }^{16}$ One could call such materials "compositionally and spatially controlled multi-scale materials". While the basic principle of LbL-assemby assures the compositional control of a composite in each layer, spray-assisted LbL-assembly at small angles or spinassisted LbL-assembly can be used to align anisotropic objects within each layer plane.

\section{CONCLUSIONS}

In summary this study shows that highly anisotropic nano-reinforced composite films composed of cellulose nanofibrils and poly(vinyl amine) can be prepared rapidly on large areas using spinassisted LbL assembly. Transparent micrometric CNF-based films with a fairly homogeneous thickness and a high level of orientation were assembled on silicon wafers using different 
rotation speeds. The in-plane orientation of CNFs in the shear flow direction was shown by studying the surface morphologies of CNF monolayers on silicon wafers along the diameter of the samples. The highest order parameter for films prepared by spin-assisted assembly was observed at sample edges for high rotation speeds. The macroscopic mechanical anisotropy of such nanocomposites films was demonstrated through a quantitative tensile characterization of free-standing (CNF/PVAm)n films both parallel and perpendicular to the nanofibril direction. Parallel and perpendicular Young's moduli determined from the experimental data were in good agreement with the theoretical ones calculated from the composite theory. Results show that this versatile and customizable LbL process provides access to nanocellulose-based materials whose mechanical strength exceeds the one of the wood (spruce) from which the nanofibrils were obtained. The work presented here concentrates on the mechanical properties of unidirectionally aligned CNF-based composites which are only one element of the complex superstructure of wood. The next steps will be the combination of several different elements to form different superstructures as schematically depicted in Figure 5B. For enabling a diversity of bio-inspired applications including sensing, packaging, electronics or optics, the preparation of nanocomposite materials and devices with anisotropic physical properties requires an extreme level of control over the positioning and alignment of nanoscale objects within the matrix material.

\section{METHODS}

Materials. Carboxymethylated nanofibrillated cellulose (CNFs, degree of substitution of 0.087 ) at a 2.5 weight percent concentration in water was provided by Innventia AB (Stockholm,

Sweden). CNF suspensions were typically prepared at a concentration of $0.9 \mathrm{~g} / \mathrm{L}$ following a procedure described in a previous work. ${ }^{19}$ Poly(vinyl amine) (trade name LUPAMIN 9095, 
PVAm, $20 \%$ in Water, $\overline{M_{w}} \approx 340000 \mathrm{~g} / \mathrm{mol}$ ) supplied for free by BASF (Ludwigshafen, Germany) was used to prepare solutions at a concentration of $1 \mathrm{~g} / \mathrm{L}$ and a pH adjusted at 8 with a solution of $\mathrm{NaOH} 0.5 \mathrm{M}$.

2" round silicon wafers (Si wafer) were obtained from Wafernet Inc. (San Jose, CA).

Substrate preparation. Si-wafer cleaning and modification (hydrophobic treatment with octadecyltrichlorosilane) were carried out using a procedure reported elsewhere..$^{19}$

LbL deposition. For dipping, we used the same experimental conditions described in a previous article for the preparation of randomly distributed cellulose-based films. ${ }^{19}$

For spin-assisted LbL assembly, the substrate was fixed in the spin coater (Laurell Technologies, North Wales, PA). The substrate was subsequently rotated at the required speed and about $0.5 \mathrm{~mL}$ of PVAm solution was added dropwise on the rotating sample followed immediately by rinsing with $1 \mathrm{~mL}$ of Milli-Q water. The substrate was then left rotating until being completely dry, monitored by the stabilization or disappearance of the interference colors. These steps were repeated for the deposition of the CNF layer. This corresponds to the deposition of one layer pair and this procedure was reiterated until the chosen thickness was attained.

A homemade dipping robot was used for the build-up of thick films, as described previously. ${ }^{19}$

For optical imaging the films were placed onto a black fabric and illuminated from above using a white lightbox. The camera is placed slightly off normal incidence, so that wafer surface reflects the lightbox surface. We calibrated the camera white balance using a piece of paper in 
similar illumination conditions. The aperture and exposition speed were automatically adjusted by the camera (Canon PowerShot G5).

Ellipsometry. The thickness measurements were performed with a spectroscopic ellipsometer SENpro (SENTECH Instruments GmbH, Berlin Germany). We used a single layer for modeling the films and fixed refractive index of 1.55 which was consistent with thicknesses obtained by AFM. No significant change of the refractive index value was observed when measured parallel and perpendicular to the direction of orientation. Each ellipsometric value is the mean of five measurements done at different positions on the sample with various orientations of the nanofibrils respectively to the laser beam.

Atomic Force Microscopy. AFM set-up and parameters used to determine the surface morphologies of CNF monolayers and the thickness of film edges are described in a previous work. ${ }^{19}$ In order to avoid that drift goes unnoticed and disturbs the orientation characterization, scanning up and down were systematically performed on each area and the images were compared.

Analysis of nanofibril orientation. The characterization of the nanofibril orientation was performed using OrientationJ a plug-in for ImageJ developed at the École Polytechnique Fédérale de Lausanne (EPFL) ${ }^{61}$ Grey scale AFM images of (PVAm/MFC) 1 with a size of $3 * 3$ $\mu \mathrm{m}$ and $15 \mathrm{~nm}$ vertical scale were loaded in ImageJ. The parameters for the orientation analysis were the following: A Gaussian window of 1 pixel to account for all the fibers (even the smallest one) and a minimum energy and coherency of $1 \%$ to exclude isotropic areas. The visualization corresponds to the superposition of the orientation direction for the color and background image 
for the contrast. The distribution of the nanofibril orientation was centered to 0 in order to calculate the order parameter.

Sample preparation for mechanical tests. Samples with a width of about $1.5 \mathrm{~mm}$ and a length of about $15 \mathrm{~mm}$ were prepared for the mechanical tests following a procedure described elsewhere. ${ }^{19}$ The positions where the samples were taken on the silicon wafers are shown in Figure 4A.

Mechanical Tests. A homemade machine for low load tests (2.5 N load cell) equipped with an environmental enclosure was used. ${ }^{62}$ A U-shape paper holder was used to support the film. Both ends of the paper support were clamped in the machine prior to the test. Once the U-shape support cut, the only bridge between the two clamps was the film. Experiments were conducted at room temperature using a constant speed rate of $0.01 \mathrm{~mm} / \mathrm{s}$ up to the film failure. The force was continuously monitored and recorded all along the tests. The reported strain and stress at break correspond to the values recorded immediately before film fracture.

Tests run at low humidity were carried out in a 2-3\% RH range atmosphere obtained thanks to a conditioned air flow. Prior to the tests, all samples were stored in a desiccator with freshly activated silica gel for $24 \mathrm{~h}$ acclimation.

The Young's modulus was measured on the first $0.3 \%$ strain of the curves. At least three sample were tested at each condition.

Digital Image Correlation. The sample deformation was recorded using digital image correlation (DIC) in order to avoid artefacts due to the compliance of the testing device. ${ }^{63,64}$ The set-up and technical procedure are identical as previously described. ${ }^{19}$ 


\section{AUTHOR INFORMATION}

Corresponding Author

*E-mail: olivier.felix@ics-cnrs.unistra.fr or decher@unistra.fr

\section{ACKNOWLEDGMENT}

The authors express their gratitude to the Ministère de l'Enseignement Supérieur, de la Recherche et de l'Innovation (MESRI), France, the Centre National de la Recherche Scientifique (CNRS), France, the International Center for Frontier Research in Chemistry, France and the Institut Universitaire de France, France for their support. We are also grateful to Christophe Contal (ICS, France) for AFM tutorial and discussions, to D. Favier and L. Jacomine from the nanomechanic platform (ICS, France) for training on mechanical tests and digital image correlation and discussions, to Innventia AB (Sweden) for supplying CNFs, to BASF (Germany) for supplying PVAm and to Lars Wågberg (KTH Royal Institute of Technology, Sweden) for constructive discussions.

\section{REFERENCES}

1 Wegst, U. G. K.; Bai, H.; Saiz, E.; Tomsia, A. P.; Ritchie, R. O. Bioinspired Structural Materials. Nat. Mater. 2015, 14, 23-36.

2 Gibson, L. J. The Hierarchical Structure and Mechanics of Plant Materials. J. Royal Soc. Interface 2012, 9, 2749-2766.

3 Ling, S.; Kaplan, D. L.; Buehler, M. J. Nanofibrils in Nature and Materials Engineering. Nat. Rev. Mater. 2018, 3, 18016. 
4 Lichtenegger, H.; Muller, M.; Paris, O.; Riekel, C.; Fratzl, P. Imaging of the Helical Arrangement of Cellulose Fibrils in Wood by Synchrotron X-Ray Microdiffraction. J. Appl. Crystallogr. 1999, 32, 1127-1133.

5 Podsiadlo, P.; Kaushik, A. K.; Arruda, E. M.; Waas, A. M.; Shim, B. S.; Xu, J.; Nandivada, H.; Pumplin, B. G.; Lahann, J.; Ramamoorthy, A.; Kotov, N. A. Ultrastrong and Stiff Layered Polymer Nanocomposites. Science 2007, 318, 80-83.

6 Bonderer, L. J.; Studart, A. R.; Gauckler, L. J. Bioinspired Design and Assembly of Platelet Reinforced Polymer Films. Science 2008, 319, 1069-1073.

7 Wang, J. F.; Cheng, Q. F.;, L. Lin; Jiang, L. Synergistic Toughening of Bioinspired Poly(vinyl Alcohol)-Clay-Nanofibrillar Cellulose Artificial Nacre. ACS Nano 2014, 8, 2739-2745.

8 Wu, M. X.; Shuai, H.; Cheng, Q.F.; Jiang, L. Bioinspired Green Composite Lotus Fibers. Angew. Chem. Int. Ed. 2014, 53, 3358-3361.

9 Klemm, D.; Kramer, F.; Moritz, S.; Lindström, T.; Ankerfors, M.; Gray, D.; Dorris, A. Nanocelluloses: A New Family of Nature-Based Materials. Angew. Chem. Int. Ed. 2011, 50, $5438-5466$.

10 Klemm, D.; Cranston, E. D.; Fischer, D.; Gama, M.; Kedzior, S. A.; Kralisch, D.; Kramer, F.; Kondo, T.; Lindström, T.; Nietzsche, S.; Petzold-Welcke, K.; Rauchfuß, F. Nanocellulose as a Natural Source for Groundbreaking Applications in Materials Science: Today's State. Mater. Today 2018, 21, 720-748. 
11 Dufresne, A.; Cavaille, J. Y.; Vignon, M. R. Mechanical Behavior of Sheets Prepared from Sugar Beet Cellulose Microfibrils. J. Appl. Polym. Sci. 1997, 64, 1185-1194.

12 Sehaqui, H.; Mushi, N. E.; Morimune, S.; Salajkova, M.; Nishino, T.; Berglund, L. A. Cellulose Nanofiber Orientation in Nanopaper and Nanocomposites by Cold Drawing. ACS Appl. Mater. Interfaces 2012, 4, 1043-1049.

13 Hakansson, K. M. O.; Fall, A. B.; Lundell, F.; Yu, S.; Krywka, C.; Roth, S. V.; Santoro, G.; Kvick, M.; Wittberg, L. P.; Wågberg, L.; Söderberg, L. D. Hydrodynamic Alignment and Assembly of Nanofibrils Resulting in Strong Cellulose Filaments. Nat. Commun. 2014, 5, 4018.

14 Torres-Rendon, J. G.; Schacher, F. H.; Ifuku, S.; Walther, A. Mechanical Performance of Macrofibers of Cellulose and Chitin Nanofibrils Aligned by Wet-Stretching: A Critical Comparison. Biomacromolecules 2014, 15, 2709-2717.

15 Geng, S.; Yao, K.; Zhou, Q.; Oksman, K. High-Strength, High-Toughness Aligned Polymer-Based Nanocomposite Reinforced with Ultralow Weight Fraction of Functionalized Nanocellulose. Biomacromolecules 2018, 19, 4075-4083.

16 Erb, R. M.; Sander, J. S.; Grisch, R.; Studart, A. R. Self-Shaping Composites with Programmable Bioinspired Microstructures. Nat. Commun. 2013, 4, 1712.

17 Decher, G. Fuzzy Nanoassemblies: Toward Layered Polymeric Multicomposites. Science 1997, 277, 1232-1237. 
18 Shim, B. S.; Zhu, J.; Jan, E.; Critchley, K.; Ho, S.; Podsiadlo, P.; Sun, K.; Kotov, N. A. Multiparameter Structural Optimization of Single-Walled Carbon Nanotube Composites: Toward Record Strength, Stiffness, and Toughness. ACS Nano 2009, 3, 1711-1722.

19 Merindol, R.; Diabang, S.; Felix, O.; Roland, T.; Gauthier, C.; Decher, G. Bio-Inspired Multiproperty Materials: Strong, Self-Healing, and Transparent Artificial Wood Nanostructures. ACS Nano 2015, 9, 1127-1136.

20 Yang, B.; Wang, L.; Zhang, M.; Lu, Z.; Tan, J.; Luo, J.; Lu, P. Water-Resistant, Transparent, Uvioresistant Cellulose Nanofiber (CNF)-Aramid Nanofiber (ANF) Hybrid Nanopaper. Mater. Lett. 2019, 240, 165-168.

21 Wassel, E.; Es-Souni, M.; Dietze, M.; Laghrissi, A.; Es-Souni, M. A Non-Fouling Multilayer Structure Based on LAPONITE®/PEG-Brushes Showing High Stiffness and Hardness. Prog. Org. Coat. 2019, 132, 108-115.

22 Wagberg, L.; Decher, G.; Norgren, M.; Lindström, T.; Ankerfors, M.; Axnäs, K. The Build-Up of Polyelectrolyte Multilayers of Microfibrillated Cellulose and Cationic Polyelectrolytes. Langmuir 2008, 24, 784-795.

23 Martin, C.; Jean, B. Nanocellulose/Polymer Multilayered Thin Films: Tunable Architectures towards Tailored Physical Properties. Nord. Pulp Pap. Res. J. 2014, 29, 19-30.

24 Podsiadlo, P.; Choi, S.-Y.; Shim, B.; Lee, J.; Cuddihy, M.; Kotov, N. A. Molecularly Engineered Nanocomposites: Layer-By-Layer Assembly of Cellulose Nanocrystals. Biomacromolecules 2005, 6, 2914-2918. 
25 Lyu, S.; Chen, Y.; Zhang, L.; Han, S.; Lu, Y.; Chen, Y.; Yang, N.; Chen, Z.; Wang, S.

Nanocellulose Supported Hierarchical Structured Polyaniline/Nanocarbon Nanocomposite Electrode via Layer-By-Layer Assembly for Green Flexible Supercapacitors. RSC Advances 2019, 9, 17824-17834.

26 Heux, L.; Chauve, G.; Bonini C. Nonflocculating and Chiral-Nematic Self-Ordering of Cellulose Microcrystals Suspensions in Nonpolar Solvents. Langmuir 2000, 16, 8210-8212.

27 Bordel, D.; Putaux, J. L.; Heux, L. Orientation of Native Cellulose in an Electric Field. Langmuir 2006, 22, 4899-4901.

28 Ebeling, T.; Paillet, M.; Borsali, R.; Diat, O.; Dufresne, A.; Cavaillé, J-Y.; Chanzy, H. Shear-Induced Orientation Phenomena in Suspensions of Cellulose Microcrystals, Revealed by Small Angle X-Ray Scattering. Langmuir 1999, 15, 6123-6126.

29 Frka-Petesic, B.; Sugiyama, J.; Kimura, S.; Chanzy, H.; Maret, G. Negative Diamagnetic Anisotropy and Birefringence of Cellulose Nanocrystals. Macromolecules 2015, 48, 8844-8857.

30 Kimura, F.; Kimura, T.; Tamura, M.; Hirai, A.; Ikuno, M.; Horii, F. Magnetic Alignment of the Chiral Nematic Phase of a Cellulose Microfibril Suspension. Langmuir 2005, 21, 2034-2037.

31 Sugiyama, J.; Chanzy, H.; Maret, G. Orientation of Cellulose Microcrystals by Strong Magnetic-Fields. Macromolecules 1992, 25, 4232-4234.

32 Cranston, E. D.; Gray, D. G. Formation of Cellulose-Based Electrostatic Layer-By-Layer Films in a Magnetic Field. Sci. Techn. Adv. Mater. 2006, 7, 319-321. 
33 Cranston, E. D.; Gray, D. G. Morphological and Optical Characterization of Polyelectrolyte Multilayers Incorporating Nanocrystalline Cellulose. Biomacromolecules 2006, $7,2522-2530$.

34 Cranston, E. D.; Gray, D. G. Birefringence in Spin-Coated Films Containing Cellulose Nanocrystals. Colloids Surf. A 2008, 325, 44-51.

35 Blell, R.; Lin, X.; Lindström, T.; Ankerfors, M.; Pauly, M.; Felix, O.; Decher, G. Generating In-Plane Orientational Order in Multilayer Films Prepared by Spray-Assisted LayerBy-Layer Assembly. ACS Nano 2017, 11, 84-94.

36 Tang, H.; Butchosa, N.; Zhou, Q. A Transparent, Hazy, and Strong Macroscopic Ribbon of Oriented Cellulose Nanofibrils Bearing Poly(ethylene Glycol). Adv. Mater. 2015, 27, 2070-2076.

37 Zhu, J.; Cao, W.; Yue, M.; Hou, Y.; Han, J.; Yang, M. Strong and Stiff Aramid Nanofiber/Carbon Nanotube Nanocomposites. ACS Nano 2015, 9, 2489-2501.

38 Cho, J.; Char, K.; Hong, J. D.; Lee, K. B. Fabrication of Highly Ordered Multilayer Films Using a Spin Self-Assembly Method. Adv. Mater. 2001, 13, 1076-1078.

39 Chiarelli, P. A.; Johal, M. S.; Casson, J. L.; Roberts, J. B.; Robinson, J. M.; Wang, H.-L. Controlled Fabrication of Polyelectrolyte Multilayer Thin Films Using Spin-Assembly. Adv. Mater. 2001, 13, 1167-1171. 
40 Kim, B. S.; Park, S. W.; Hammond, P. T. Hydrogen-Bonding Layer-By-Layer Assembled Biodegradable Polymeric Micelles as Drug Delivery Vehicles from Surfaces. ACS Nano 2008, 2, 386-392.

41 Patro, T. U.; Wagner, H. D. Layer-By-Layer Assembled PVA/Laponite Multilayer FreeStanding Films and Their Mechanical and Thermal Properties. Nanotechnology 2011, 22, 455706.

42 Zimnitsky, D.; Shevchenko, V. V.; Tsukruk, V. V. Perforated, Freely Suspended LayerBy-Layer Nanoscale Membranes. Langmuir 2008, 24, 5996-6006.

43 Landel, R. F.; Nielsen, L. E. Mechanical Properties of Polymers and Composites, Second Edition; Taylor and Francis: NewYork, 1993, p 580.

44 Rezakhaniha, R.; Agianniotis, A.; Schrauwen, J. T. C.; Griffa, A.; Sage, D.; Bouten, C. V. C.; van de Vosse, F. N.; Unser, M.; Stergiopulos, N. Experimental Investigation of Collagen Waviness and Orientation in the Arterial Adventitia Using Confocal Laser Scanning Microscopy. Biomech. Model. Mechanobiol. 2012, 11, 461-473.

45 LeMieux, M. C.; Roberts, M.; Barman, S.; Jin, Y. W., Kim, J. M.; Bao, Z., Self-Sorted, Aligned Nanotube Networks for Thin-Film Transistors. Science 2008, 321, 101-104.

46 Benitez, A. J.; Torres-Rendon, J.; Poutanen, M.; Walther, A. Humidity and Multiscale Structure Govern Mechanical Properties and Deformation Modes in Films of Native Cellulose Nanofibrils. Biomacromolecules 2013, 14, 4497-4506. 
47 Ward, I. M.; Sweeney, J. An Introduction to the Mechanical Properties of Solid Polymers, Second edition; Wiley: Chichester, 2004, p 394.

48 Eichhorn, S. J.; Young, R. J. The Young's Modulus of a Microcrystalline Cellulose. Cellulose 2001, 8, 197-207.

49 Hepworth, D. G.; Bruce, D. M. A Method of Calculating the Mechanical Properties of Nanoscopic Plant Cell Wall Components from Tissue Properties. J. Mater. Sci. 2000, 35, $5861-5865$.

50 Šturcová, A.; Davies, G. R.; Eichhorn, S. J. Elastic Modulus and Stress-Transfer Properties of Tunicate Cellulose Whiskers. Biomacromolecules 2005, 6, 1055-1061.

51 Iwamoto, S.; Kai, W.; Isogai, A.; Iwata, T. Elastic Modulus of Single Cellulose Microfibrils from Tunicate Measured by Atomic Force Microscopy. Biomacromolecules 2009, $10,2571-2576$.

52 Tanaka, F.; Iwata, T. Estimation of the Elastic Modulus of Cellulose Crystal by Molecular Mechanics Simulation. Cellulose 2006, 13, 509-517.

53 Azzam, F.; Chaunier, L.; Moreau, L. C.; Lourdin, D.; Bertoncini, P.; Cathala, B. Relationship between Young's Modulus and Film Architecture in Cellulose Nanofibril-Based Multilayered Thin Films. Langmuir 2017, 33, 4138-4145.

54 Henriksson, M.; Berglund, L. A.; Isaksson, P.; Lindström, T.; Nishino, T. Cellulose Nanopaper Structures of High Toughness. Biomacromolecules 2008, 9, 1579-1585. 
55 Yang, W.; Bian, H.; Jiao, L.; Wu, W.; Deng, Y.; Dai, H. High wet-Strength, Thermally

Stable and Transparent TEMPO-Oxidized Cellulose Nanofibril Film via Cross-Linking with Poly-Amide Epichlorohydrin Resin. RSC Adv. 2017, 7, 31567-31573.

56 Mohammadi, P.; Toivonen, M. S.; Ikkala, O.; Wagermaier, W.; Linder, M. B. Aligning Cellulose Nanofibril Dispersions for Tougher Fibers. Sci.Rep. 2017, 7, 11860.

57 Tucker III, C. L.; Liang, E. Stiffness Predictions for Unidirectional Short-Fiber Composites: Review and Evaluation. Compos. Sci. Technol. 1999, 59, 655-671.

58 Song, J.; Chen, C.; Zhu, S.; Zhu, M.; Dai, J.; Ray, U.; Li, Y.; Kuang, Y.; Li, Y.; Quispe, N.; Yao, Y.; Gong, A.; Leiste, U. H.; Bruck, H. A.; Zhu, J. Y.; Vellore, A.; Li, H.; Minus, M. L.; Jia, Z.; Martini, A. et al. Processing Bulk Natural Wood into a High-Performance Structural Material. Nature 2018, 554, 224-228.

59 Zhu, M.; Wang, Y.; Zhu, S.; Xu, L.; Jia, C.; Dai, J.; Song, J.; Yao, Y.; Wang, Y.; Li, Y.; Henderson, D.; Luo, W.; Li, H.; Minus, M. L.; Li, T.; Hu, L. Anisotropic, Transparent Films with Aligned Cellulose Nanofibers. Adv. Mater. 2017, 29, 1606284.

60 Mittal, N.; Ansari, F.; Gowda, V. K.; Brouzet, C.; Chen, P.; Larsson, P. T.; Roth, S. V.; Lundell, F.; Wågberg, L.; Kotov, N. A.; Söderberg, L. D. Multiscale Control of Nanocellulose Assembly: Transferring Remarkable Nanoscale Fibril Mechanics to Macroscale Fibers. ACS Nano 2018, 12, 6378-6388.

61 Rezakhaniha, R.; Fonck, E.; Genoud, C.; Stergiopulos, N. Role of Elastin Anisotropy in Structural Strain Energy Functions of Arterial Tissue. Biomech. Model. Mechanobiol. 2011, 10, 599-611. 
62 Gauthier, C.; Schirrer, R. Time and Temperature Dependence of the Scratch Properties of Poly(methylmethacrylate) Surfaces. J. Mater. Sci. 2000, 35, 2121-2130.

63 Chu, T. C.; Ranson, W. F.; Sutton, M. A. Applications of Digital-Image-Correlation Techniques to Experimental Mechanics. Exp. Mech. 1985, 25, 232-244.

64 Hild, F.; Roux, S. Digital Image Correlation: From Displacement Measurement to Identification of Elastic Properties - A Review. Strain 2006, 42, 69-80.

SYNOPSIS
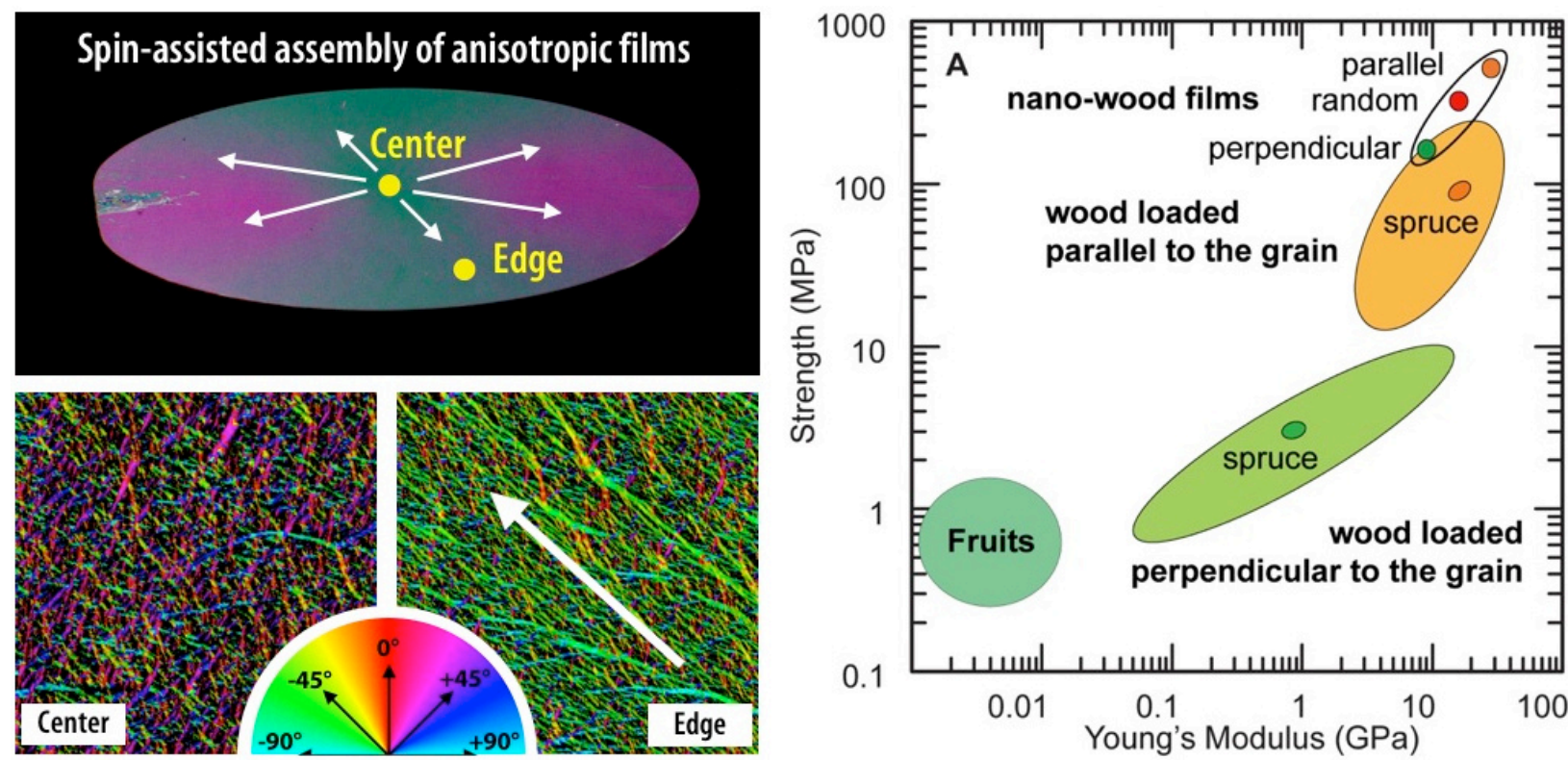